\title{
RELACIÓN ENTRE EL ESTILO DE APRENDIZAJE Y LA PROFESIÓN ELEGIDA POR ESTUDIANTES UNIVERSITARIOS ${ }^{1}$
}

\section{María Matalinares Calvet}

La investigación que se presenta tuvo como objetivo establecer si existe o no relación entre el estilo de aprendizaje y la profesión elegida por estudiantes universitarios. Para tal efecto, se evaluó a 1,036 estudiantes, de ambos sexos cuyas edades fluctuaban entre los 16 y 28 años de diferentes facultades de la UNMSM a quienes se aplicó el Inventario de Estilos de Aprendizaje de Kolb. Los resultados muestran que hay una asociación sistemática entre las categorías de la variable estilo de aprendizaje con determinadas categorías de profesión. Lo que significaría que la probabilidad de estudiar determinada profesión no es independiente al estilo de aprendizaje. De igual modo se halló que las variables sexo y edad, están asociadas con el estilo de aprendizaje.

PALABRAS CLAVE: Estilos de aprendizaje, profesión.

The present study aimed to establish if learning styles related to the career chosen by university students. One thousand thirty six students, both male and female, aged 16 to 28 , from different faculties of San Marcos National Major University were assessed with Kolb Inventory of Learning Styles. Outcomes show a systematic association among categories of learning style variable and some categories of career variable. This would mean that probability to study a defined career is not independent from learning style. Associations among sex and age variables and learning style were also found.

KEY WORDS: Learning style, career.

${ }^{1}$ Miembrosde la investigación: Carlos Arenas y Alejandro Dioses. 


\section{INTRODUCCIÓN}

Anualmente postula a la Universidad Nacional Mayor de San Marcos una enorme cantidad de jóvenes que desean hacerse profesionales. El número de estos es superior al de cualquier otra universidad en el Perú. En efecto, el año 2000 postularon 66, 392 alumnos e ingresaron 5,672; en el 2001 60,394 e ingresaron 6,507 y en el 2002 postularon más de 65,000 estudiantes e ingresaron 6,500. Sin embargo en 2000 egresaron 2,404 y el 2001 egresaron 2,152 estudiantes, lo cual demuestra que menos del $50 \%$ del número de jóvenes que ingresan a la Universidad egresan anualmente. No obstante hace 7 años, la situación era algo distinta, la proporción de egresantes era mayor que la que actualmente se tiene (Oficina General de Planificación, 2001).

Ahora bien, es evidente que este fenómeno tiene en su base una serie de factores, los cuales no han sido aún suficientemente investigados pese a su indudable importancia.

Por otro lado, las profesiones más elegidas en la UNMSM son: Medicina Humana (6,284 postulantes), Derecho y Ciencia Política (5,240 postulantes) Ingeniería de Sistemas (4,580 postulantes) mientras que las menos elegidas son Física (105 postulantes) Química (141 postulantes) y Filosofía (144 postulantes). Pero no se puede asegurar que todos los que postulan a tales carreras poseen las características intelectuales, afectivas, personales y físicas que exige el estudio y práctica de la profesión.

Sobre la cuestión vocacional se han ocupado autores como Calle (1972) quien hizo un análisis de Las motivaciones Vocacionales en un grupo Universitario. Tal autor señaló que la vocación se manifiesta como una tendencia al ejercicio de determinada ocupación y el estilo de vida que ella conlleva, estando integrada por diversos elementos y procesos psicológicos entre los que la motivación ocupa un lugar destacado. Asimismo agregó que debemos entender la motivación como aquel factor que despierta mantiene y dirige el comportamiento en el sentido de un objetivo y/o meta. El propósito de la investigación fue determinar las diversas características que presenta la motivación vocacional y las preguntas que busca responder se refieren a los objetivos de la elección, el tiempo de elección, el conocimiento de la profesión elegida y la satisfacción vocacional. El método utilizado fue descriptivo transversal. El instrumento utilizado fue el Cuestionario Vocacional, que consta de 12 ítems que se relacionan con motivos vocacionales, humanitarios, instrumentales, teóricos, aptitudinales, económicos, egoístas y sociales. La muestra estuvo compuesta por alumnos del primeros años del ciclo diurno de las Facultades de Derecho, Educación y Letras de la UNMSM., la Facultad de Medicina de la Universidad Cayetano Heredia y la facultad de Matemáticas y Petróleo de la Universidad Nacional de Ingeniería. Los resultados de esta investigación demuestran que la mayoría precisa motivos vocacionales, en segundo lugar motivos humanistas y en seguida motivos aptitudinales y teóricos. Los motivos sociales adquieren menor significación en la muestra. La mayoría declara haber efectuado su elección vocacional durante la educación secundaria y entre los 18 y 19 años en promedio. Las cualidades que se requieren para el ejercicio de las profesiones examinadas son en orden de importancia: los rasgos de personalidad, las aptitudes intelectuales, las características físicas y las cualidades morales. Los estudiantes que evidencian mayor conocimiento sobre los fines de su carrera son los de Educación y los que poseen menor conocimiento son los de Psicología. Los estudiantes de todos los grupos esperaban una satisfacción personal en el ejercicio de su profesión destacando sobre las otras que son: altruista, teórica, economía y social.

Córdova y Flores (1974) en una investigación sobre el análisis de los intereses vocacionales de un grupo de estudiantes secundarios de diferente nivel socioeconómico 
encontraron que los llamados intereses vocacionales manifestados por los individuos, constituyen una de las bases donde se apoya la consejería en la elección de una carrera. Son conceptos inherentes a la definición de interés vocacional: la atención, satisfacción, persistencia, preferencia y otras más que pueden ser invocadas. Entre los factores determinantes de los intereses se encuentran las diferencias sexuales, los determinantes culturales, la posición económica social. Este último factor es tomado preeminentemente en esta investigación descriptiva de diseño transversal. El instrumento utilizado para el proceso de recogida de datos es el Inventario de Intereses de Angelini en la versión castellana de F. del Olmo. La muestra estuvo compuesta por alumnos del cuarto y quinto año de educación secundaria común diurna de ambos sexos, cuyas edades fluctuaban entre los 15 y 18 años, pertenecientes a Colegios Estatales y Particulares de Lima Metropolitana. Los colegios seleccionados fueron exclusivamente para mujeres o para varones y representaban a su vez a tres niveles de condición socioeconómica Alta, Media y Baja. Los intereses inventariados de los estudiantes secundarios revelaron diferencias según el nivel socioeconómico. Entre los varones se encontró diferencias significativas al $5 \%$ en las áreas de Ciencias Físicas, Ciencias Biológicas, Calculo, Organización Burocrática, Servicio Social y Literatura. Las mujeres presentan diferencias significativas a15\% en las áreas de Ciencias Físicas, Ciencias Biológicas, Calculo, Organización Burocrática, Servicio Social, Literatura y Musical. El grupo de varones de todos los niveles socioeconómico revelan preferencias mayores por las Ciencias Físicas y Biológicas, las mujeres por su lado revelan motivaciones mayores por el área de Servicio Social.

Tradicionalmente la orientación vocacional se ocupaba de los aspectos de la personalidad, aptitudes, y habilidades intelectuales de los postulantes, pero no se tomaba en cuenta aspectos importantes inherentes al aprendizaje.

Por otro lado, en el área de la Cognición una variable que cada vez cobra más importancia en los estudios y en la actividad pedagógica, así como la psicología educativa, está constituida por los estilos de aprendizaje.

Pero ¿Como aparece la idea de estilo de aprendizaje? Bonham (1987), a semejanza de Keefe (1979) y Dunn y Dunn (1978), nos remontan a los años sesenta para ubicar la aparición del interés de los investigadores por la idea de Estilo de aprendizaje. En búsqueda de palabras más exactas para definir las diferencias individuales de los alumnos, los investigadores desarrollan conceptos útiles (definiciones, modelos) y prácticos (instrumentos, técnicas) para medir las características de los alumnos en términos de aprendizaje, más que en términos generales de funcionamiento cognitivo. La noción de estilo cognitivo no es muy específica y se restringe al contexto del laboratorio, por otro lado la noción de estilo de aprendizaje aparece en un momento en que los investigadores han querido responder a las necesidades de adaptar el modo de presentar los cursos y el material pedagógico, a las necesidades particulares de los estudiantes. Por lo tanto ahí reside la diferencia fundamental entre el estilo cognitivo y el estilo de aprendizaje, esta última tiene una perspectiva esencialmente pedagógica que la primera no presenta.

Según Bonham (1987), la noción de estilo de aprendizaje ha podido surgir gracias a cuatro influencias que han contribuido a su valoración:

1. La investigación sobre la mejor modalidad sensorial a privilegiar en la enseñanza

2. El acento puesto sobre el individuo, como resultado de la educación progresiva y de la investigación sobre los estilos cognitivos.

3. La identificación de las diferencias individuales entre los grupos en función de variables tales como la edad y el sexo, así como la voluntad política de responden a \}as necesidades de los, niños excepcionales.

4. La educación de los adultos y la noción de formación profesional continúa. 
La mayoría de las corrientes ideológicas en relación con los estilos de aprendizaje se plasman durante los años sesenta en diferentes instrumentos. La reflexión sobre las diferencias individuales más importantes a tener en cuenta en el contexto escolar se orienta en muchas direcciones. La diversidad conceptual que caracteriza a la noción de estilo cognitivo toma rápidamente amplitud, aún en el caso del estilo de aprendizaje. Para todos los autores se trata de tener en cuenta los factores que tienen participación en los procesos de aprendizaje de los alumnos y generan las diferencias individuales importantes $\mathrm{y}$ pertinentes para los alumnos que desean obtener un mejor aprendizaje. Muchas topologías han sido desarrolladas.

Es en la última década en la que más se ha señalado lo importante de considerar los estilos de aprendizaje de los alumnos en el proceso de aprendizaje; algunos investigadores superponen los significados de estilos de aprendizaje, estilos cognitivos, estrategias de aprendizaje, aunque concuerdan en señalar que cada individuo interviene de manera activa en su aprendizaje, captando, elaborando y utilizando la información de acuerdo con sus características individuales (Alonso, Gallego y Honey, 1994, Askew, 2000; Cano y Justicia,1996) Coloma y Tafur, 2000;Gonzáles-Pienda, 1996; Kogan,1981).

"Los estilos de aprendizaje son los rasgos cognitivos, afectivos y fisiológicos, que sirven como indicadores relativamente estables, de cómo los discentes perciben, interaccionan y responden a sus ambientes de aprendizaje (Keefe, 1988; citado por Alonso,1994). Es el conjunto de hábitos, formas o estilos de cada persona para actuar o pensar en cada situación. Cómo tendemos a tomar decisiones en distintas situaciones y entornos, bajo condiciones de incertidumbre, en situaciones límite, en estados de serenidad, etc.

En este sentido Kolb ha planteado un modelo de cuatro etapas: Observación y Reflexión, Experiencias Concretas, Conceptualización Abstracta y Experimentación Activa. Partiendo de estas cuatro etapas del aprendizaje experiencial se han identificado cuatro capacidades necesarias para lograr un aprendizaje eficaz.

La primera es la capacidad de Experiencia Concreta (Ee) que consiste en que el sujeto debe ser capaz de poner énfasis en la interacción con otras personas haciendo un uso mayor de sus propias sensaciones al enfrentarse a los problemas que le toca vivir. La segunda es la Capacidad de Observación Reflexiva (OR) y está referida a la capacidad del sujeto para entender las ideas y situaciones desde diferentes puntos de vista. Para ello debe hacer uso de objetividad y de juicio cuidadoso, manejando además los propios pensamientos y sentimientos para llegar a formarse una opinión. La tercera capacidad es la Conceptualización Abstracta (CA) que se caracteriza porque el sujeto debe ser capaz de utilizar la lógica y las ideas para entender y comprender los problemas y situaciones que se presentan. Debe usar un planteamiento sistemático y recurrir a ideas y concepciones teóricas con la finalidad de resolver problemas. La última Capacidad, es la de Experimentación Activa (EA). Ello supone que el sujeto asume un enfoque práctico con relación a los problemas, con una mayor preocupación respecto a la forma como funcionan las cosas, sin tomar en cuenta la observación ni el análisis de los problemas.

Según Kolb, las personas tienen desarrolladas en forma desigual las cuatro capacidades, predominando dos respecto a los otras dos. Las diferencias entre ellas permiten definir cada uno de los estilos. En coherencia con ello, Kolb ha planteado la existencia de cuatro estilos de aprendizaje que son: convergente, divergente, asimilador y acomodador.

Según Kolb en el Estilo Convergente hay predominio de la conceptualización abstracta y de la experimentación activa; sujetos con este estilo muestran habilidad por resolver problemas y tomar decisiones; son buenos en la aplicación práctica de las ideas y en situaciones donde hay más de una contestación; no son emotivos, prefieren las cosas a las personas, manifiestan intereses étnicos. En el Estilo Divergente se ha desarrollado más la 
experiencia concreta y la organización reflexiva; sujetos con este estilo tienen la capacidad de involucrarse en experiencias novedosas, llevan a cabo experimentos y proyectos, destacan por su imaginación. Pueden ver las situaciones desde diferentes perspectivas, son emotivos y se interesan por la gente. En el Estilo Asimilador existe un mayor desarrollo de la conceptualización abstracta y la observación reflexiva, además de la capacidad de desarrollar modelos teóricos y hacer uso del razonamiento inductivo; les preocupa más los conceptos que las personas, y están menos interesados en el uso práctico de las teorías.

Por último, en el Estilo Acomododador el desarrollo de la experimentación activa y la experiencia concreta son determinantes. Sujetos con este estilo utilizan muy poco el análisis lógico, se basan en su intuición y en un análisis técnico, muestran habilidad para llevar a cabo planes, orientados a la acción, les gustan nuevas experiencias, son arriesgados, se adaptan a las circunstancias inmediatas, es característico de individuos en el área de los Negocios (Robles, 2002).

El tema de los Estilos de Aprendizaje, se está constituyendo cada vez más en objeto de investigación. Han investigado sobre el tema en Puerto Rico, Santos, Pagan y Alvarez (1995) quienes realizaron una evaluación sobre el efecto del estilo cognoscitivo y la presencia o ausencia de dislexia en la comprensión lectora en 60 niños cuyas edades fluctuaban entre los ocho y doce años, de tercero a quinto grados elementales de San Juan de Puerto Rico. Los resultados sugerían que tanto niños disléxicos como no disléxicos pueden mejorar su comprensión si son inducidos a un estilo cognoscitivo reflexivo. En México Gonzáles, Castañeda y Maytorena (2000) estudiaron el efecto de los estilos de aprendizaje en el rendimiento académico en estudiantes universitarios cuya edad promedio era de 19 años: los resultados sólo explicaron el 7\% de aprovechamiento académico.

En Canadá Aarón y Skakun (1999) correlacionaron las características de los estudiantes con sus estilos de aprendizaje y su comienzo en la carrera de medicina. Utilizaron el Inventario de Aproximación y Estudio de Habilidades (ASSIT). Los resultados de cada uno fue correlacionado con los pre-requisitos de los Puntajes Promedios de los Grados, Números de años de Experiencia Pre-médica y puntajes sobre su autobiografía, entrevistas y cartas de referencia. Se encontró que un alto índice de los puntajes de estudiantes se correlacionaban significativamente con los estudiantes más jóvenes que ingresaron al colegio de médicos, y con altos puntajes en sus promedios. Lo que llevaba a una correlación positiva entre los puntajes promedios y los perfiles estudiantiles en un grupo de estudiantes con más de cuatro años de experiencia pre-médica.

En el Perú (1989) Eisler realizó una adaptación de la segunda versión del Inventario de Estilos de Aprendizaje de Kolb en 518 alumnos entre 16 y 26 años de diversos niveles socioeconómicos. Los resultados señalan la validez de constructo, de contenido y la confiabilidad del instrumento. Los resultados del grupo fueron homogéneos, aunque los contadores registraron mayor actividad que los economistas. De otro lado se encontró que a mayor tiempo de estudios hay mayor tendencia hacia la reflexión.

Escurra (1991) realizó una adaptación a la primera versión del IEA de Kolb en 250 estudiantes de psicología de dos universidades limeñas, encontrando que la conceptualización abstracta es más desarrollada que la experimentación activa. Encontró además que en lo alumnos de UNMSM hay un estilo de aprendizaje asimilador y de la PUCP no hay uno definido, predominando el de la experiencia activa.

Paz (1998) relacionó los estilos de aprendizaje, estilos de enseñanza y rendimiento académico en estudiantes de secundaria basado en el modelo de Dunn y Dunn. Los resultados mostraron que existe una correlación positiva entre el estilo de aprendizaje y la motivación, persistencia y responsabilidad.

Luján (2000) en una investigación sobre estilos de aprendizaje, inteligencia y rendimiento escolar en estudiantes de quinto de secundaria encontró que el estilo de 
aprendizaje prevalente fue el divergente y el menos frecuente el convergente.

Delgado, Ponce, Bulnes, Escurra y Pequeña (2000) en una investigación sobre necesidad cognitiva y aprendizaje de estudiantes universitarios encontraron altos niveles de necesidad cognitiva en los varones, siendo el estilo de aprendizaje divergente el más frecuente en la muestra estudiada.

Schmidt (2001) encontró que el estilo de aprendizaje más frecuente en los estudiantes universitarios de la carrera de Psicología es el divergente y el menos frecuente es el convergente. También demostró que existen diferencias significativas en el área de Observación, Reflexión únicamente entre el primer con cuarto, sexto y décimo ciclo y quinto con sétimo ciclo y existen diferencias significativas en el área de Experimentación Activa entre primer con sétimo, octavo, noveno, décimo, segundo con sétimo, octavo, noveno y décimo ciclo, tercero con sétimo, octavo, décimo, cuarto con sétimo y décimo ciclo y quinto con sétimo y décimo, y por último encontró que no existe diferencia significativas entre las áreas de los estilos de aprendizaje en función al sexo.

Otro estudio interesante es el de Capella, Col ama, Manrique, Quevedo, Revilla, Tafur y Vargas (2002) quienes han abordado el tema de los Estilos de Aprendizaje en los estudiantes de la Pontificia Universidad Católica del Perú que cursaban en el semestre académico 2001-1 el primer o tercer año de estudios con la finalidad de conocer si la permanencia en la universidad influye sobre estos estilos de aprendizaje.

Si bien es cierto que la investigación acerca de los estilos de aprendizaje viene desarrollándose, en la dirección de encontrar relaciones con otras variables, no se han realizado trabajos en los que se relacione con la profesión elegida por los jóvenes universitarios y esto es muy importante, ya que como se ha dicho líneas arriba, hay un enorme vacio en el conocimiento del tema, el cual involucra a todos los estudiantes universitarios del Perú, toda vez que a la universidad de San Marcos acceden estudiantes de los más diversos sectores sociales, culturales y económicos de la población peruana y por consiguiente constituye una muestra razonablemente representativa de lo que ocurre a nivel nacional. Ahora bien, una elección profesional mal hecha tiene evidente consecuencias negativas tanto para el desarrollo de la ciencia y tecnología del país como para sus posibilidades de desarrollo económico. Asimismo, para lo que se ha dado por llamar capital humano. Es en este contexto que se ha formulado el siguiente el problema:

¿Existe relación entre la profesión elegida y el estilo de aprendizaje de los estudiantes universitarios?

\section{OBJETIVOS}

El objetivo general es establecer si existe o no relación entre el estilo de aprendizaje y la profesión elegida por estudiantes universitarios.

Los Objetivos Específicos aluden a:

1) Realizar un diagnóstico de los estilos de aprendizaje que caracterizan a los estudiantes de las diferentes facultades de la UNMSM.

2) Correlacionar los Estilos de aprendizaje con las profesiones elegidas por los estudiantes cuya profesión coincide con su preferencia vocacional.

Este trabajo tiene su impacto en dos sentidos: de un lado, en el aspecto teórico, porque brinda elementos para la discusión acerca de la Teoría de Kolb en lo concerniente a los estilos de aprendizaje. Por otro lado, contribuye al cuestionamiento y mejora de la orientación profesional y ocupacional que debe hacerse a los jóvenes en el Perú, y que debe tomar en cuenta sus estilos de aprendizaje.

En síntesis, el trabajo tiene impacto teórico y práctico y sus resultados podrían beneficiar a un amplísimo sector de la juventud peruana; por lo menos aquellos que accedan a la Universidad. 
De otro lado, es importante, porque los trabajos acerca de los estilos de aprendizaje en el Perú, apenas si se han iniciado, a pesar de que constituye un tópico importante en el terreno de la cognición.

Por consiguiente este estudio brinda información importante en estas dos vertientes y evidentemente contribuye de modo muy notable en el terreno de la Psicología Educativa y de la Cognición en particular. Más aún, si se tiene en cuenta, que dentro de la política y Lineamientos de investigación de la facultad de psicología, se reconoce el papel central de los procesos psicológicos en todas y cada una de las diferentes situaciones y niveles de los procesos de aprendizaje y la demanda de elaborar el diagnóstico y evaluación de los principales problemas educativos peruanos.

La hipótesis de investigación fue: «Existe relación entre los estilos de aprendizaje planteados por Kolb y las profesiones elegidas por los universitarios».

\section{METODO}

En concordancia con la hipótesis formulada, la metodología empleada puede definirse del siguiente modo: Se empleó el método descriptivo con un diseño descriptivo correlacional.

\section{Sujetos}

Se ha tomado aleatoriamente 1,036 estudiantes de ambos sexos, cuyas edades fluctuaban entre los 16 y 28 años, que cursaban entre el ler y 8vo ciclo de estudios de diferentes facultades de la UNMSM. En la muestra existen estudiantes con alto, bajo y mediano promedio ponderado. Las variables principales del estudio son: Estilos de aprendizaje y profesión elegida.

\section{Técnicas e Instrumentos}

Se ha utilizado la técnica de la observación indirecta, dado el carácter de los fenómenos estudiados. El Instrumento utilizado fue el Inventario de Estilos de Aprendizaje (IEA) de Kolb. A este Instrumento Miguel Escurra realizó un adaptación. El instrumento tiene validez de contenido por criterio de jueces; el IEA posee validez de construcción y por 10 tanto el constructo psicológico de Kolb es válido en nuestro medio. El inventario tiene confiabilidad y consistencia interna (Escurra, 1991).

El Inventario de Estilos de Aprendizaje, contiene palabras que fueron seleccionadas como las más aceptables socialmente con cada una de las fases del ciclo de aprendizaje experiencial. De total de palabras se seleccionan solo treinta y seis (36), de las cuales veinticuatro (24) están asociados a cada una de las cuatro fases del ciclo de aprendizaje experiencial; las doce (12) palabras restantes son incluidas como elementos distractores con la finalidad de controlar la deseabilidad social, por ello no son utilizados para el cómputo final. De modo que, los veinticuatro (24) palabras evalúan las cuatro etapas de aprendizaje experiencial, como son: Experiencia Concreta (EC), Observación Reflexiva (OR), Conceptualización Abstracta (CA), Experimentación Activa (EA).

Estas etapas son denominadas también modalidades de aprendizaje y conforman además las dimensiones concreta-abstractas y actividades-reflexión. Estas treinta y seis (36) palabras están ordenadas en nueve (9) grupos de cuatro (4) palabras cada grupo, de manera que los sujetos deben responder ordenando jerárquicamente cada grupo de palabras según el grado con el cual se percibe a la palabra que mejor caracterice su estilo individual de aprender, asignando los puntajes que van de uno al cuatro (1-4), del menos al más característico.

\section{Procedimiento de recogida de datos}

1. Coordinación con las autoridades de las diferentes facultades de la universidad.

2. Determinación de la muestra. 
3. Preparación y reproducción de los instrumentos a usarse.

4. Evaluación de la muestra con el IEA de Kolb. La recolección de los datos fue en forma colectiva en grupos no mayores de 28 personas y cuidando que los alumnos comprendan las instrucciones y asuman compromiso con la veracidad de de sus respuestas.

5. Calificación de las Pruebas

\section{Técnicas de Procesamiento de datos}

En la presente investigación se hace uso de las tablas de frecuencias, para describir los resultados. Para la Contrastación d e hipótesis se utiliza la prueba no parámetrica Chi Cuadrada, debido a que los datos con que se trabajan son de nivel nominal. También se usaron medidas de asociación según Coeficiente Phi y Coeficiente V de Cramer. Así como el análisis cualitativo y discusión de los resultados en base a los antecedentes de investigación y la teoría de Kolb.

\section{RESULTADOS}

A continuación se exponen los resultados del trabajo:

Tabla 1. Frecuencia de la muestra en los Estilos de Aprendizaje.

\begin{tabular}{|l|l|l|}
\hline Estilos de aprendizaje & Frecuencia & Percentil \\
\hline Convergente & 259 & 25.0 \\
Acomodador & 264 & 25.5 \\
Asimilador & 276 & 26.6 \\
Divergente & 237 & 22.9 \\
Total & 1036 & 100.0 \\
\hline
\end{tabular}

\section{Estilo de aprendizaje}

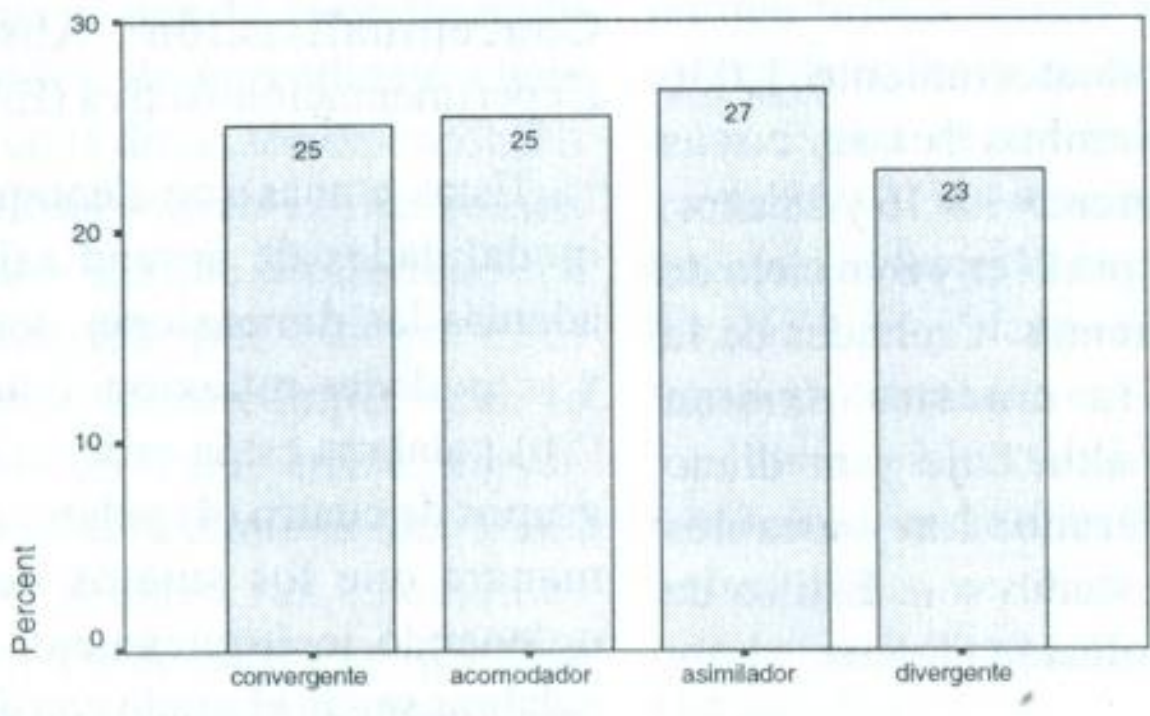

Estilo de aprendizaje 
Como pucde apreciarse en la tabla 1 y $25.5 \% \quad(264)$ en el estilo acomodador, un fig.l el 25\% de la muestra (259) se $26.6 \%(276)$ en el estilo asimilikfor $y$ un encuentran ubicados en el estiloconvergente, $22.9 \%$ (237) se ubica en el estilo divergente,

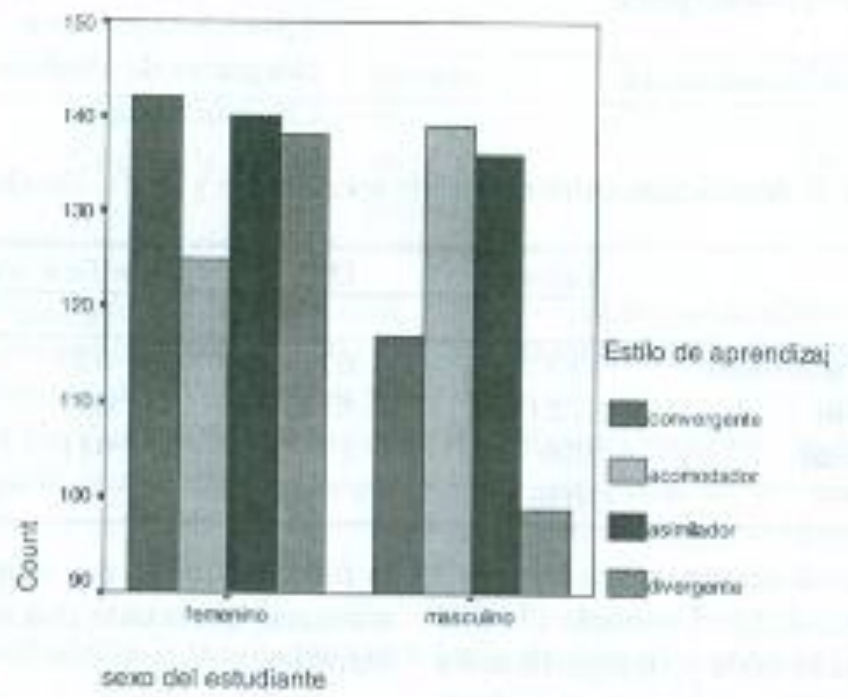

Fig. 2. Distribución de la muestra según las variables estilo de aprendizaje y sexo.

Como puede observarse en la fig. 2 puede observarse que en las mujeres el estilo de aprendizaje más caracteristico es el convergente y en los varones el acomcxdador es el más predominante. Ahora bien, el estito de aprendizaje acomodador es menos frecuente en las mujeres y el divergente es el menos frecuente en los varones.

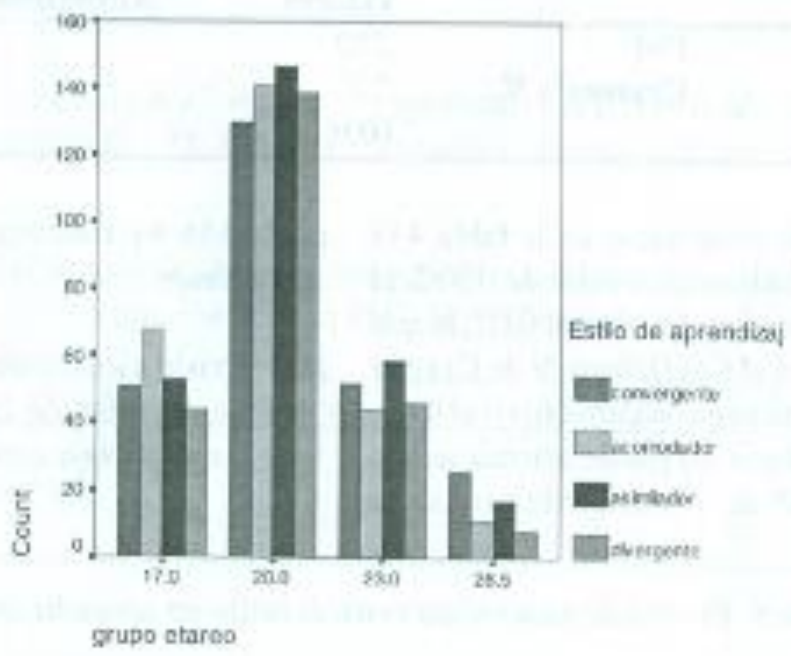

Fig. 3. Distribución de la muestra en la variable estilu de aprendizaje segun el grupo etáreo.

Como puede ibservarse en la Fig. 3 en el grupo etáreo que va de 16 a 18 años el estilo más frecuente es el acomomodador. De los 19 a los 21 el estilo asimilador e el másfrecuente. El grupo que va de 22 a 24 años el más frecuente es el asimilador. En el último grupo etáreo que va de 25 a 28 años el estilo mas frecuente es el convergente. 


\section{Contraste de Hipótesis}

H 1: Existe asociación sistemática entre las categorías de la variable Estilo de Aprendizaje con determinadas categorías de Profesión a la luz de la Chi cuadrada.

Tabla 3. Asociación entre estilos de aprendizaje y profesión elegida.

\begin{tabular}{|l|l|l|l|}
\hline & Valores & Dif. & Significación asintótica (2 lados) \\
\hline Pearson Chi & 159.598 & 63 & .000 \\
cuadrado & 172.005 & 63 & .000 \\
Likelihood Ratio & .006 & 1 & .941 \\
Asociación lineal & 1036 & & \\
$\mathrm{~N}^{\circ}$ de casos & & & \\
\hline
\end{tabular}

Como puede observarse en la tabla 3 el valor estadístico de Chi Cuadrado 159.598 es superior al de la tabla y su significación asociada es de 0.000 por lo cual se rechaza la hipótesis nula, por consiguiente puede afirmarse que existe una relación entre las variables.

\section{Tabla 4. Medidas de Asociación según Coeficiente Phi y Coeficiente V de Cramer entre estilos de aprendizaje y profesión elegida.}

\begin{tabular}{|l|l|l|l|}
\hline & & \multicolumn{1}{|c|}{ Valores } & Aproximación Significación \\
\hline Nominal & Phi & .392 & .000 \\
Nominal & Cramer's & .227 & .000 \\
$\mathrm{~N}^{\circ}$ de casos & V. & 1036 & \\
\hline
\end{tabular}

Como puede observarse en la tabla 4 el Coeficiente Phi obtuvo un valor de 0.392, el cual es altamente significativo al 0.01 , lo que se corrobora con el Coeficiente $\mathrm{V}$ de Cramer 0.227 que es altamente significativo al 0.01. Lo que implica que no puede afirmarse que la probabilidad de estudiar determinada profesión es independiente al estilo de aprendizaje.

H2: Existe asociación sistemática entre las categorías de la variable estilos de aprendizaje con el sexo.

Tabla 5. Prueba de asociación entre el estilo de aprendizaje y el sexo.

\begin{tabular}{|l|l|l|l|}
\hline & Valores & Dif. & $\begin{array}{l}\text { Significación } \\
\text { asintótica (2 lados) }\end{array}$ \\
\hline Pearson Chi cuadrado & 6.835 & 3 & .077 \\
Likelihood Ratio & 6.849 & 3 & .077 \\
Asociación lineal & .790 & 1 & .374 \\
$\mathrm{~N}^{\circ}$ de casos & 1036 & & \\
\hline
\end{tabular}

Como puede observarse en la tabla 5 el valor estadístico Chi Cuadrado 6.835 es significativo al 0.077 de nivel confianza. Por lo cual se rechaza la hipótesis nula. 
Tabla 6. Medidas de Asociación según el coeficiente Phi y el coeficiente U de Cramer.

\begin{tabular}{|l|l|l|l|}
\hline & & Valores & $\begin{array}{l}\text { Aproximación } \\
\text { Significación }\end{array}$ \\
\hline Nominal & Phi & .081 & .077 \\
Nominal & Cramer's V. & .0 .81 & .077 \\
$\mathrm{~N}^{\circ}$ de casos & & 1036 & \\
\hline
\end{tabular}

Como puede observarse en la tabla 6 el Coeficiente Phi obtuvo un valor de 0.081 el cual es significativo al 0.077, lo que se corrobora con el Coeficiente V de Cramer 0.081 que es significativo al 0.077. Lo que implica que no puede afirmarse que el estilo de aprendizaje es independiente al sexo.

H3: Existe asociación sistemática entre las categorías de la variable estilos de aprendizaje con la edad.

Tabla 7. Chi cuadrado de la asociación entre estilo de aprendizaje y edad

\begin{tabular}{|l|l|l|l|} 
& \multicolumn{1}{|c|}{ Valores } & Dif. & Significación asintótica (2 lados) \\
\hline Pearson Chi cuadrado & 75.402 & 33 & .000 \\
Likelihood Ratio & 77.265 & 33 & .000 \\
Asociación lineal & .613 & 1 & .434 \\
$\mathrm{~N}^{\circ}$ de casos & 1036 & & \\
\hline
\end{tabular}

Como puede observarse en la tabla 7 el valor estadístico chi cuadrado 75.402 es significativo al 0.000 de nivel confianza. Por lo cual se rechaza la hipótesis nula.

Tabla 8. Medidas de asociación entre las variables estilo de aprendizaje y edad según coeficiente Phi y V de Cramer.

\begin{tabular}{|l|l|l|l|}
\hline & & Valores & Aproximación Significación \\
\hline Nominal & Phi & .270 & .000 \\
Nominal & Cramer's V. & .156 & .000 \\
$\mathrm{~N}^{\circ}$ de casos & & 1036 & \\
\hline
\end{tabular}

Como puede observarse en la tabla 8 el coeficiente Phi obtuvo un valor de 0.270 el cual es significativo al 0.000 , lo que se corrobora con el Coeficiente V de Cramer 0.156 que es significativo al 0.000. Lo que implica que no puede afirmarse que el estilo de aprendizaje es independiente a la edad. 


\section{DISCUSIÓN}

Los resultados expuestos, presentan el siguiente panorama: En primer término se ha comprobado que los cuatro estilos de aprendizaje referidos por Kolb se encuentran en proporciones más o menos similares en la muestra (véase tabla $1 \mathrm{y}$ fig. 1) hallazgos que coinciden con los reportados por Eisler (1989). Esto quiere decir que entre los estudiantes de la Universidad de San Marcos los cuatro estilos tenderían ha distribuirse equitativamente.

Dicho en otros términos, el 25\% (convergente) de la población sanmarquina se caracteriza por tener un predominio de la conceptualización abstracta y de la experimentación activa; tener habilidad para resolver problemas y tomar decisiones, además de ser buenos en la aplicación práctica de las ideas. Otro 25.5\% (acomodador) en cambio se caracteriza por el desarrollo de la experimentación activa y la experiencia concreta son determinantes. Según Robles (2002) sujetos con este estilo utilizan muy poco el análisis lógico, se basan en su intuición y en un análisis técnico, muestran habilidad para llevar a cabo planes, orientados a la acción, buscan nuevas experiencias, son arriesgados, se adaptan a las circunstancias inmediatas.

El $26.6 \%$ de la población, tendería a ser asimilador y por consiguiente tendería a un mayor desarrollo de la conceptualización abstracta y la observación reflexiva, además de poseer la capacidad de desarrollar modelos teóricos y hacer uso del razonamiento inductivo; les preocupa más los conceptos que las personas, y están menos interesados en el uso práctico de las teorías. Finalmente, el $22.9 \%$ que ostenta el estilo divergente ha desarrollado más la experiencia concreta y la organización reflexiva; estos sujetos tendrían la capacidad de involucrarse en experiencias novedosas, llevar a cabo experimentos y proyectos, destacar por su imaginación. Pueden ver las situaciones desde diferentes perspectivas.

Pero al penetrar con mayor minuciosidad en la distribución de los estilos de aprendizaje, se ha podido establecer que en ingeniería industrial, arte, comunicación social, filosofía, bibliotecología, lingüística e ingeniería eléctrica el estilo de aprendizaje más frecuente es el acomodador. Mientras que en medicina, química, derecho, bioquímica, matemáticas y geografía el estilo asimilador es el más frecuente. En carreras como economía, educación, trabajo social, sociología y contabilidad el estilo convergente es el más frecuente y por último en biología, historia y antropología el estilo divergente es el más frecuente. No obstante quedaría por aclarar -y estudios posteriores deben hacerlo- si tales estilos en las referidas carreras están asociados con el rendimiento académico deseado. De momento solo se puede notar que de las 22 carreras estudiadas en siete, el estilo más frecuente es el acomodador; en seis de ellas, el asimilador en cinco el estilo convergente y en tres el divergente.'

Al relacionar la variable sexo con el estilo de aprendizaje, se ha encontrado que entre las mujeres predomina el estilo convergente y en los varones el acomodador. Paradójicamente en las mujeres el estilo menos frecuente es precisamente el acomodador, en tanto que en los varones el divergente es el menos frecuente (veáse fig.3) Lo cual significaría que las mujeres tienden a ser más abstractas y a resolver problemas y tomar decisiones. Mientras que los varones utilizarían menos el análisis lógico y más la intuición.

Por lo demás, los hallazgos aquí expuestos, se condicen con los reportes de Escurra (1991) quien encontró que el estilo asimilador predominaba entre los estudiantes de San Marcos. No obstante, nuestros hallazgos (veáse tabla 1) muestran que hay una mínima diferencia porcentual $(1.1 \%)$ entre el asimilador y el estilo acomodador que ocupa el segundo lugar; y aún entre este y los que ocupan el tercero y cuarto lugar, la diferencia porcentual es relativamente pequeña. Más adelante volveremos sobre el punto al contrastar 
la hipótesis correspondiente. Sin embargo es notable que lo aquí reportado difiere de los hallazgos de Luján (2000) Delgado, Ponce, Bulnes, Escurra y Pequeña (2000) quienes reportaron la predominancia del estilo divergente.

Es conveniente detenerse ahora en los aspectos inferenciales del tratamiento estadístico. De las tres hipótesis de trabajo sometidas a prueba, en todos los casos se ha rechazado la hipótesis nula y se ha aceptado la alterna; aunque con distinto nivel de confianza (veáse tablas 3-8).

Pero, ¿Qué significa esto en términos cualitativos?

En primer lugar se ha establecido que sí hay relación estadística entre el estilo de aprendizaje y la profesión elegida. Es decir, que la probabilidad de elegir una profesión, no es independiente del estilo de aprendizaje del que elige. Este hallazgo, tiene enorme importancia, pues mostraría la necesidad de tomar en cuenta la variable estilos de aprendizaje cuando se hacen elecciones profesionales y por tanto cuando se hace la orientación en este sentido. Ello pondría al tapete el tema del proceso de orientación vocacional y profesional, ya que consecuentemente con los resultados aquí expuestos, el psicólogo que hace orientación vocacional debería evaluar esta variable y considerar los resultados, en la fase de toma de decisiones.

En lo concerniente a la relación con la variable sexo, se ha encontrado que efectivamente esta variable guarda también relaciones estadísticas con los estilos de aprendizaje, aun cuando el nivel de confianza es menor (véase tabla 5). Ello supone que el ser varón o mujer implicaría la probabilidad de que predomine un estilo distinto (fig. 2). Por lo demás, los resultados que aquí se discuten, pondrían en debate la muy difundida tendencia de creer que las mujeres son más intuitivas y los varones más racionales. Los hallazgos aquí expuestos mostrarían precisamente lo contrario; lo cual podría constituir un indicador de que las condiciones de educación y de vida de la mujer están cambiando. Aunque es posible también que la tradicional caracterización de la mujer como mas intuitiva y menos racional haya estado más basada en prejuicios que en observaciones válidas y confiables). Aunque es importante señalar que estos resultados difieren a los hallazgos de Schmidt quien no encontró diferencias significativas entre las áreas de los estilos de aprendizaje en función al sexo.

En lo concerniente a la edad y su relación con los estilos de aprendizaje, se ha podido demostrar que también en este caso las variables se hallan asociadas; lo cual implica que el pertenecer a uno u otro grupo etáreo, por lo menos aumenta la probabilidad de poseer uno u otro estilo de aprendizaje, (veáse fig.3; tablas 7 y 8) En efecto, puede apreciarse que entre 16 y 18 años el estilo más frecuente es el acomodador. En los dos subgrupos siguientes (de 19 a 21 y de 22 a 24 años) el estilo asimilador es el más frecuente. En el último grupo etáreo que va de 25 a 28 años el estilo más frecuente es el convergente. Esto ratificaría la idea de que los Estilos cognitivos no son estables y que van variando en función de las experiencias y necesidades de los individuos.

Efectivamente, en este sentido Keefe (1988) citado por Revilla (2002) sostiene que los estilos de aprendizaje no son inamovibles, son relativamente estables, es decir, que pueden cambiar. Los alumnos conforme avanzan en su proceso de aprendizaje descubren mejores formas o modos de aprender, por lo tanto, van a variar su estilo, además dependerá de la circunstancias, contextos y tiempos de aprendizaje que tengan que enfrentar.

En suma se ha comprobado que la variable estilos de aprendizaje se halla asociada con una serie de variables y que es pertinente establecer tales relaciones, no solo como resultado de los intereses científicos, sino también con fines prácticos. 


\section{CONCLUSIONES}

1. En estudiantes de la UNMSM los cuatro estilos de aprendizaje se hallan presentes de modo equitativo. Lo cual implica que no se puede afirmar la predominancia general de un estilo sobre los otros aún cuando se observa mayor frecuencia simple en el estilo acomodador.

2. Al agrupar los datos por edad se ha establecido que esta variable no es irrita con respecto al estilo de aprendizaje, que en el grupo de menor edad tiende a ha predominar el estilo divergente y en el de mayor edad el estilo convergente, mientras que en los dos grupos etáreos intermedios predomina el estilo asimilador.

3. Se ha comprobado también que los varones y las mujeres difieren en su estilo de aprendizaje. Predominando en la mujeres el estilo convergente y en los varones el estilo acomodador. Por consiguiente el sexo resulta una variable importante en la dominancia de uno u otro estilo.

4. Finalmente se ha establecido que el tipo de carrera elegida guarda relación con el estilo de aprendizaje del que elige. Ello permite suponer que el tipo de carrera elegida no es independiente del estilo de aprendizaje.

\section{REFERENCIAS BIBLIOGRÁFICAS}

Aaron, S. y Skankun, E. (1999). Correlación entre estilos de aprendizaje y rendimiento en estudiantes de medicina: uned.es/ departamento/301/acop-rop.6htm.

Alonso, C.; Gallego, D. y Honey, P. (1994). Los estilos de aprendizaje. Bilbao: Mensajero.

Askew, M. (2000). Cinco modelos de estilos de aprendizaje. members.tripod.com/ elhogarI200012000-10/cinco-modelosde-estilos-de-aprendizaje.htm

Calle, M. (1972). Análisis de Las motivaciones vocacionales en un grupo universitario. Tesis para optar el Grado de Bachiller en Psicología, Lima: UNMSM.

Cano, F. y Justicia, F. (1996). Los estilos de aprendizaje en psicología y educación. En 1. Gonzáles-Pienda, Escoriza, 1. Gonzáles, R. y Barca, A. (Eds). Psicología de la Instrucción. Componentes cognitivos y afectivos del aprendizaje escolar Vol.2 Barcelona: EUB.

Capella, J., Coloma, C., Manrique, L., Quevedo, E., Revilla, D., Tafur, R. y Vargas, J. (2002) Estilos de Aprendizaje en los estudiantes de la Pontificia Universidad Católica del Perú// jcapell@ puc.edu.pe

Chevrier, 1. ; Fortin, G. Théberg,M. y Leblanc, R. Estilos de aprendizaje: Una Perspectiva Histórica. Investigadores las universidades: Québec a Hull, Université St-Paul, Université d'Ottawa respectivamente. Traducción de Juan Yaringaño.

Coloma, C. y Tafur, R. (2000). Sobre los estilos de enseñanza y aprendizaje. Educación. 9( 17), 51-79.

Cordova, A. y Flores, C. (1974). Análisis de los intereses vocacionales de un grupo de estudiantes secundarios de diferente nivel socioeconómico. Tesis para optar el Grado de Bachiller en Psicología, UNMSM, Lima.

Delgado, A.; Ponce, c.; Bulnes, M.; Escurra, M. y Pequeña, J. (2000). Relación entre necesidad cognitiva y estilos de aprendizaje en estudiantes de la UNMSM. Revista de Investigación en Psicología. 3(2), 9-18.

Eisler, S. (1989). Adaptación de un instrumento: El inventario de Estilos de Aprendizaje. Memoria para optar el grado de Bachiller en Humanidades con mención en Psicología 
PUCP, Lima.

Escurra, M. (1991). Adaptación del Inventario de estilos de Aprendizaje de Kolb en estudiantes de psicología pertenecientes a diferentes universidades de Lima Metropolitana. Tesis para optar el título profesional de psicólogo. UNMSM, Lima.

Gonzáles, D.; Castañeda, S. y Maytorena, M. (2000). Estilos de aprendizaje y aprovechamiento en ingresantes universitarios. Revista de Psicología de la PUCP,18(2), 199-225.

Gonzáles-Pienda,J. (1996). Estilos cognitivos y de aprendizaje. En Gonzáles-Pienda, J.; Escoriza, J.; Gonzáles, R. y Barca, A. (eds). Psicología de la Instrucción. Componentes cognitivos y afectivos del aprendizaje escolar, Vol 2. Barcelona:EUB.

Kogan, N. (1981). Las implicaciones de los estilos cognoscitivos en la educación. En La psicología en la práctica educativa. México: Trillas.

Luján, E. (1999). Estilos de Aprendizaje considerando la inteligencia y el rendimiento escolar en alumnos de quinto año de secundaria de Colegios de educación tradicional y educación alternativa. Tesis para optar el grado de Magíster en Psicología con Mención Psicología Educativa. UNMSM, Lima.

Robles, A. Estilos de aprendizaje. httpll www.galeon.com.aprender a aprender general indice

Santos, D. Pagán, N. y Alvarez, A. (1995).

Comprensión de la lectura como función del estilo cognoscitivo y la condición de dislexia. Revista Interamericana de Psicología, 29(2), 191-200.

Schmidt, J. (2001). Estilos de Aprendizaje en estudiantes de psicología de la Universidad de San Martín de Porres. Tesis para optar el Título de Licenciado en Psicología de la UPSMP. Lima. 